\title{
SOME $q$-CONGRUENCES ARISING FROM CERTAIN IDENTITIES
}

\author{
CHEN WANG AND HE-XIA NI*
}

\begin{abstract}
In this paper, by constructing some identities, we prove some $q$-analogues of some congruences. For example, for any odd integer $n>1$, we show that

$$
\begin{gathered}
\sum_{k=0}^{n-1} \frac{\left(q^{-1} ; q^{2}\right)_{k}}{(q ; q)_{k}} q^{k} \equiv(-1)^{(n+1) / 2} q^{\left(n^{2}-1\right) / 4}-(1+q)[n] \quad\left(\bmod \Phi_{n}(q)^{2}\right), \\
\sum_{k=0}^{n-1} \frac{\left(q^{3} ; q^{2}\right)_{k}}{(q ; q)_{k}} q^{k} \equiv(-1)^{(n+1) / 2} q^{\left(n^{2}-9\right) / 4}+\frac{1+q}{q^{2}}[n] \quad\left(\bmod \Phi_{n}(q)^{2}\right),
\end{gathered}
$$

where the $q$-Pochhanmmer symbol is defined by $(x ; q)_{0}=1$ and $(x ; q)_{k}=(1-x)(1-x q) \cdots(1-$ $\left.x q^{k-1}\right)$ for $k \geq 1$, the $q$-integer is defined by $[n]=1+q+\cdots+q^{n-1}$ and $\Phi_{n}(q)$ is the $n$-th cyclotomic polynomial. The $q$-congruences above confirm some recent conjectures of $\mathrm{Gu}$ and Guo.
\end{abstract}

\section{INTRODUCTION}

In 2010, Sun and Tauraso [11] studied some congruence properties of sums concerning central binomial coefficients $\left(\begin{array}{c}2 k \\ k\end{array}\right)$ where $k \in \mathbb{N}=\{0,1, \ldots\}$. For example, let $p$ be an odd prime and $r \in \mathbb{Z}^{+}$, they proved that for any $m \in \mathbb{Z}$ with $p \nmid m$,

$$
\sum_{k=0}^{p^{r}-1} \frac{\left(\begin{array}{c}
2 k \\
k+d
\end{array}\right)}{m^{k}} \equiv u_{p^{r}-|d|}(m-2) \quad(\bmod p),
$$

where $|d| \in\left\{0, \ldots p^{r}\right\}$ and the sequence of polynomials $u_{n}(x)(n \in \mathbb{N})$ is defined as follows:

$$
u_{0}(x)=0, u_{1}(x)=1 \text {, and } u_{n+1}(x)=x u_{n}(x)-u_{n-1}(x)(n=2,3, \ldots) .
$$

In particular, they obtained that

$$
\sum_{k=0}^{p^{r}-1} \frac{\left(\begin{array}{c}
2 k \\
k
\end{array}\right)}{2^{k}} \equiv(-1)^{\left(p^{r}-1\right) / 2} \quad(\bmod p) .
$$

Later, Sun [10] further proved that (1.1) also holds modulo $p^{2}$.

Throughout the paper, the $q$-integer $[n]_{q}$ is defined as $[n]=[n]_{q}=1+q+\ldots+q^{n-1}$, while the $q$-pochhammer symbol ( $q$-shifted factorial) is defined by $(x ; q)_{0}=1$ and $(x ; q)_{k}=$

2020 Mathematics Subject Classification. Primary 11A07, 11B65; Secondary 05A30, 05A10.

Key words and phrases. $q$-congruences, $q$-Pochhammer symbol, cyclotomic polynomial, $q$-identities.

*Corresponding author. 
$(1-x)(1-x q) \cdots\left(1-x q^{k-1}\right)$ for $k \geq 1$. And recall that the $n$-th cyclotomic polynomial $\Phi_{n}(q)$ is defined as

$$
\Phi_{n}(q)=\prod_{\substack{1 \leq k \leq n \\ \operatorname{gcd}(n, \bar{k})=1}}\left(q-\zeta^{k}\right),
$$

where $\zeta$ is an $n$-th primitive root of unity.

As we all know, identities or congruences usually have nice $q$-analogues. In recent years, $q$-analogues of identities and congruences have been investigated by various authors (cf. for example, [1, 2, 3, 4, 5, 6, 7, 8, 9, 12, 13]). In 2010, Guo and Zeng 8] gave the following $q$-analogue of (1.1):

$$
\sum_{k=0}^{n-1} \frac{\left(q ; q^{2}\right)_{k}}{(q ; q)_{k}} q^{k} \equiv(-1)^{(n-1) / 2} q^{\left(n^{2}-1\right) / 4} \quad\left(\bmod \Phi_{n}(q)\right),
$$

where $n$ is a positive odd integer. Morever, Guo [7] established the following generalization of (1.2):

$$
\sum_{k=0}^{n-1} \frac{\left(q ; q^{2}\right)_{k}}{(q ; q)_{k}} q^{k} \equiv(-1)^{(n-1) / 2} q^{\left(n^{2}-1\right) / 4} \quad\left(\bmod \Phi_{n}(q)^{2}\right) .
$$

It should be pointed out that (1.3) for odd primes $n$ was first conjectured by Tauraso [13] in 2013.

Recently, Gu and Guo [3] provided some $q$-congruences formally analogous to (1.2) by making use of Carlitz's transformation formula (cf. 2]). For any odd integer $n>1$, they proved that

$$
\begin{aligned}
& \sum_{k=0}^{n-1} \frac{\left(q^{-1} ; q^{2}\right)_{k}}{(q ; q)_{k}} q^{k} \equiv(-1)^{(n+1) / 2} q^{\left(n^{2}-1\right) / 4} \quad\left(\bmod \Phi_{n}(q)\right), \\
& \sum_{k=0}^{n-1} \frac{\left(q^{3} ; q^{2}\right)_{k}}{(q ; q)_{k}} q^{k} \equiv(-1)^{(n+1) / 2} q^{-(n-3)^{2} / 4} \quad\left(\bmod \Phi_{n}(q)\right) .
\end{aligned}
$$

Our first theorem concerns the generalization of (1.4).

Theorem 1.1. For any odd integer $n>1$ we have

$$
\begin{gathered}
\sum_{k=0}^{n-1} \frac{\left(q^{-1} ; q^{2}\right)_{k}}{(q ; q)_{k}} q^{k} \equiv(-1)^{(n+1) / 2} q^{\left(n^{2}-1\right) / 4}-(1+q)[n] \quad\left(\bmod \Phi_{n}(q)^{2}\right), \\
\sum_{k=0}^{n-1} \frac{\left(q^{-1} ; q^{2}\right)_{k}}{(q ; q)_{k}} q^{2 k} \equiv(-1)^{(n+1) / 2} q^{\left(n^{2}+3\right) / 4}-2 q[n] \quad\left(\bmod \Phi_{n}(q)^{2}\right) .
\end{gathered}
$$

Letting $q \rightarrow 1$ in (1.6) or (1.7) we obtain the following congruence.

Corollary 1.1. Let $p$ be an odd prime and $r \in \mathbb{Z}^{+}$. Then

$$
\sum_{k=0}^{p^{r}-1} \frac{1}{2^{k}(2 k-1)}\left(\begin{array}{c}
2 k \\
k
\end{array}\right) \equiv(-1)^{(p-1) / 2}+2 p^{r} \quad\left(\bmod p^{2}\right) .
$$


Remark 1.1. (1.6) and (1.8) were conjectured by Gu and Guo in [3]. (1.7) is actually a different $q$-analogue of (1.8).

$\mathrm{Gu}$ and Guo [3] also attempted to find a mod $\Phi_{n}(q)^{2}$ extension of (1.5) but failed. The next theorem gives a different $q$-analogue of (1.1) and generalizes (1.5).

Theorem 1.2. Let $n>1$ be an odd integer. Then

$$
\begin{aligned}
& \sum_{k=0}^{n-1} \frac{\left(q ; q^{2}\right)_{k}}{(q ; q)_{k}} q^{2 k} \equiv(-1)^{(n-1) / 2} q^{\left(n^{2}-5\right) / 4}+\frac{q-1}{q}[n] \quad\left(\bmod \Phi_{n}(q)^{2}\right), \\
& \sum_{k=0}^{n-1} \frac{\left(q^{3} ; q^{2}\right)_{k}}{(q ; q)_{k}} q^{k} \equiv(-1)^{(n+1) / 2} q^{\left(n^{2}-9\right) / 4}+\frac{1+q}{q^{2}}[n] \quad\left(\bmod \Phi_{n}(q)^{2}\right) .
\end{aligned}
$$

Letting $q \rightarrow 1$ we have the following corollary which confirms [3, (1.8)].

Corollary 1.2. Let $p$ be an odd prime and $r \in \mathbb{Z}^{+}$. Then

$$
\sum_{k=0}^{p^{r}-1} \frac{(2 k+1)}{2^{k}}\left(\begin{array}{c}
2 k \\
k
\end{array}\right) \equiv(-1)^{(p+1) / 2}+2 p^{r} \quad\left(\bmod p^{2}\right) .
$$

Remark 1.2. (1.10) is essentially an extension of (1.5). In fact, noting that $q^{n} \equiv 1\left(\bmod \Phi_{n}(q)\right)$, we immediately get

$$
q^{\left(n^{2}-9\right) / 4} \equiv q^{-(n-3)^{2} / 4} \quad\left(\bmod \Phi_{n}(q)\right) .
$$

Differently from Gu and Guo's method, we will not use Carlitz's transformation. Our strategy is to find some new identities linking the $q$-congruences to be solved with (1.3). Assume that $F(k, q)$ is a rational function in $q$ such that $F(k, q) / F(k-1, q)$ can be written as a ratio of two polynomials in $q$. Now we want to find a polynomial $R(k, q)$ such that $\sum_{k=0}^{n} F(k, q) R(k, q)$ has a closed form. Consider the summation $\sum_{k=0}^{n} F(k, q)$. By the definition of $F$, we may write

$$
\frac{F(k, q)}{F(k-1, q)}=\frac{S(k, q)}{T(k, q)}
$$

or equivalently,

$$
F(k, q) T(k, q)=F(k-1, q) S(k, q),
$$

where $S(k, q)$ and $T(k, q)$ are polynomials of $q$. Then summing both sides of (1.12) from $k=1$ to $n$ and via some simple computation we find that

$$
\sum_{k=0}^{n}(T(k, q)-S(k+1, q)) F(k, q)=F(0, q) T(0, q)-F(n, q) S(n+1, q) .
$$

Here $T(k, q)-S(k+1, q)$ is the polynomial that we hope to find.

The proofs of Theorem 1.1 and 1.2 will be given in Sections 2 and 3 respectively. 


\section{Proof of Theorem 1.1}

Lemma 2.1. For any positive integer n, we have the following identities

$$
\sum_{k=0}^{n-1} \frac{\left(q ; q^{2}\right)_{k}}{(q ; q)_{k}} q^{k}+\frac{1}{1-q} \sum_{k=0}^{n-1} \frac{\left(q^{-1} ; q^{2}\right)_{k}}{(q ; q)_{k}} q^{k}\left(1-q^{k}\right)=\frac{\left(q ; q^{2}\right)_{n-1}}{(q ; q)_{n-1}} q^{n-1}
$$

and

$$
\frac{1}{1-q} \sum_{k=0}^{n-1} \frac{\left(q^{-1} ; q^{2}\right)_{k}}{(q ; q)_{k}} q^{k}\left(q-q^{k}\right)=-\frac{\left(q ; q^{2}\right)_{n-1}}{(q ; q)_{n-1}}
$$

Proof. Set

$$
F_{1}(k, q)=\frac{\left(q^{-1} ; q^{2}\right)_{k}}{(q ; q)_{k}} q^{k}
$$

It is easy to verify that

$$
\left(1-q^{k}\right) F_{1}(k, q)=q\left(1-q^{2 k-3}\right) F_{1}(k-1, q) .
$$

Summing both sides of (2.3) from $k=1$ to $n-1$ and noting that the left-hand side of (2.3) vanishes when $k=0$, we arrive at

$$
\sum_{k=0}^{n-1}\left(1-q^{k}\right) F_{1}(k, q)=\sum_{k=1}^{n-1} q\left(1-q^{2 k-3}\right) F_{1}(k-1, q)=\sum_{k=0}^{n-2} q\left(1-q^{2 k-1}\right) F_{1}(k, q),
$$

or equivalently,

$$
\sum_{k=0}^{n-1} q\left(1-q^{2 k-1}\right) F_{1}(k, q)-\sum_{k=0}^{n-1}\left(1-q^{k}\right) F_{1}(k, q)=q\left(1-q^{2 n-3}\right) F_{1}(n-1, q) .
$$

Then (2.1) follows by noting that

$$
q\left(1-q^{2 k-1}\right) F_{1}(k, q)=(q-1) \frac{\left(q ; q^{2}\right)_{k}}{(q ; q)_{k}} q^{k}
$$

for all $k$ among $0,1, \ldots, n-1$.

To show (2.2) we set

Now we find that

$$
F_{2}(k, q)=\frac{\left(q^{-1} ; q^{2}\right)_{k}}{(q ; q)_{k}}
$$

$$
\left(1-q^{k}\right) F_{2}(k, q)=\left(1-q^{2 k-3}\right) F_{2}(k-1, q) .
$$

Then we may obtain (2.2) by some similar arguments as above.

Lemma 2.2. For any odd integer $n>1$, we have

$$
\frac{\left(q ; q^{2}\right)_{n-1}}{(q ; q)_{n-1}} \equiv-q[n] \quad\left(\bmod \Phi_{n}(q)^{2}\right) \text {. }
$$


Proof. Clearly,

$$
\frac{\left(q ; q^{2}\right)_{n-1}}{(q ; q)_{n-1}}=\frac{(q ; q)_{2 n-2}}{(-q ; q)_{n-1}(q ; q)_{n-1}^{2}} .
$$

Note that

$$
q^{n} \equiv 1 \quad\left(\bmod \Phi_{n}(q)\right)
$$

and

$$
q^{j} \not \equiv 1 \quad\left(\bmod \Phi_{n}(q)\right) \quad \text { for all } j=1,2, \ldots, n-1 .
$$

Thus we have

$$
\frac{(q ; q)_{2 n-2}}{(q ; q)_{n-1}^{2}}=\left(1-q^{n}\right) \frac{\prod_{j=1}^{n-2}\left(1-q^{n+j}\right)}{\prod_{j=1}^{n-1}\left(1-q^{j}\right)} \equiv \frac{1-q^{n}}{1-q^{-1}}=-q[n] \quad\left(\bmod \Phi_{n}(q)^{2}\right) .
$$

By [1, Corollary 10.2.2(c)] we have

$$
(-q ; q)_{n-1}=\frac{(-q ; q)_{n}}{1+q^{n}} \equiv \frac{1}{2} \sum_{k=0}^{n} \frac{(q ; q)_{n} q^{n(n+1) / 2}}{(q ; q)_{k}(q ; q)_{n-k}} \equiv 1 \quad\left(\bmod \Phi_{n}(q)\right) .
$$

Substituting (2.6) and (2.7) into (2.5) we immediately obtain the desired lemma.

Proof of Theorem 1.1. We first prove (1.6). Combining (2.1) and (2.2) and with the help of Lemma 2.2 we obtain that

$$
\begin{aligned}
\sum_{k=0}^{n-1} \frac{\left(q ; q^{2}\right)_{k}}{(q ; q)_{k}} q^{k}+\sum_{k=0}^{n-1} \frac{\left(q^{-1} ; q^{2}\right)_{k}}{(q ; q)_{k}} q^{k} & =\frac{\left(q ; q^{2}\right)_{n-1}}{(q ; q)_{n-1}}\left(1+q^{n-1}\right) \\
& \equiv-q[n]\left(1+q^{-1}\right) \\
& =-(1+q)[n] \quad\left(\bmod \Phi_{n}(q)^{2}\right)
\end{aligned}
$$

Now (1.6) follows from (1.3).

With the help of (2.2), we have

$$
q \sum_{k=0}^{n-1} \frac{\left(q^{-1} ; q^{2}\right)_{k}}{(q ; q)_{k}} q^{k}-\sum_{k=0}^{n-1} \frac{\left(q^{-1} ; q^{2}\right)_{k}}{(q ; q)_{k}} q^{2 k}=(q-1) \frac{\left(q ; q^{2}\right)_{n-1}}{(q ; q)_{n-1}} .
$$

Then we obtain (1.7) by noting (1.6) and Lemma 2.2.

The proof of Theorem 1.1 is now complete.

\section{Proof of Theorem 1.2}

Lemma 3.1. For any positive integer $n$ we have the following identities.

$$
(1-q) \sum_{k=0}^{n-1} \frac{\left(q^{3} ; q^{2}\right)_{k}}{(q ; q)_{k}} q^{k}-\frac{1}{q} \sum_{k=0}^{n-1} \frac{\left(q ; q^{2}\right)_{k}}{(q ; q)_{k}} q^{k}\left(1-q^{k}\right)=\frac{\left(q^{3} ; q^{2}\right)_{n-1}}{(q ; q)_{n-1}}\left(q^{n-1}-q^{n}\right)
$$


and

$$
\sum_{k=0}^{n-1} \frac{\left(q ; q^{2}\right)_{k}}{(q ; q)_{k}} q^{k}\left(1-q^{k+1}\right)=(1-q) \frac{\left(q^{3} ; q^{2}\right)_{n-1}}{(q ; q)_{n-1}}
$$

Proof. Set

$$
G_{1}(k, q)=\frac{\left(q ; q^{2}\right)_{k}}{(q ; q)_{k}} q^{k}
$$

Then we may easily check that

$$
\left(1-q^{k}\right) G_{1}(k, q)=\left(q-q^{2 k}\right) G_{1}(k-1, q) .
$$

Summing both sides of the above identity from $k=1$ to $n-1$ we have

$$
q \sum_{k=0}^{n-1}\left(1-q^{2 k+1}\right) G_{1}(k, q)-\sum_{k=0}^{n-1}\left(1-q^{k}\right) G_{1}(k, q)=\left(q-q^{2 n}\right) G_{1}(n-1, q) .
$$

Thus we obtain (3.1) by noting

$$
(1-q)\left(q^{3} ; q^{2}\right)_{k}=\left(1-q^{2 k+1}\right)\left(q ; q^{2}\right)_{k}
$$

for all $k$ among $0,1, \ldots, n-1$.

Also, (3.2) can be deduced by setting

$$
G_{2}(k, q)=\frac{\left(q ; q^{2}\right)_{k}}{(q ; q)_{k}}
$$

and noting that

$$
\left(1-q^{k}\right) G_{2}(k, q)=\left(1-q^{2 k-1}\right) G_{2}(k-1, q) .
$$

Proof of Theorem 1.2. By (3.2) we have

$$
\sum_{k=0}^{n-1} \frac{\left(q ; q^{2}\right)_{k}}{(q ; q)_{k}} q^{k}-q \sum_{k=0}^{n-1} \frac{\left(q ; q^{2}\right)_{k}}{(q ; q)_{k}} q^{2 k}=\left(1-q^{2 n-1}\right) \frac{\left(q ; q^{2}\right)_{n-1}}{(q ; q)_{n-1}}
$$

Then (1.9) follows from (1.3) and Lemma 2.2. Substituting (1.3) and (1.9) we immediately get $(1.10)$.

The proof of Theorem 1.2 is now complete.

Acknowledgments. The first author is supported by the National Natural Science Foundation of China (Grant No. 11971222). 


\section{REFERENCES}

[1] G. Andrews, R. Askey and R. Roy, Special Functions, Cambridge Univ. Press, Cambridge, 1999.

[2] L. Carlitz, A q-identity, Fibonacci Quart. 12 (1974), 369-372.

[3] C.-Y. Gu and V.J.W. Guo, Two q-congruences from Carlitz's formula, Period. Math. Hungar., to appear.

[4] V.J.W. Guo, A q-analogue of a Ramanujan-type supercongruence involving central binomial coefficients, J. Math. Anal. Appl. 458 (2018), 590-600.

[5] V.J.W. Guo, A q-analogue of the (J.2) supercongruence of Van Hamme, J. Math. Anal. Appl. 466 (2018), $766-788$.

[6] V.J.W. Guo, A q-analogue of the (I.2) supercongruence of Van Hamme, Int. J. Number Theory 15 (2019), $29-36$.

[7] V.J.W. Guo, Proof of a q-congruence conjectured by Tauraso, Int. J. Number Theory 15 (2019), 37-41.

[8] V.J.W. Guo and J. Zeng, Some congruences involving central q-binomial coefficients, Adv. Appl. Math. 45 (2010) 303-316.

[9] H.-X. Ni and H. Pan, Divisibility of some binomial sums, Acta Arith., to appear.

[10] Z.-W. Sun, Binomial coefficients, Catalan numbers and Lucas quotients, Sci. China Math. 53 (2010), $2473-2488$.

[11] Z.-W. Sun and R. Tauraso, New congruences for central binomial coefficients, Adv. Appl. Math. 45 (2010), $125-148$.

[12] R. Tauraso, q-Analogs of some congruences involving Catalan numbers, Adv. in Appl. Math. 48 (2010), 603-614.

[13] R. Tauraso, Some q-analogs of congruences for central binomial sums, Colloq. Math. 133 (2013), 133-143.

(Chen Wang) Department of Mathematics, Nanjing University, Nanjing 210093, People's RePUblic OF CHINA

E-mail address: cwang@smail.nju.edu.cn

(He-Xia Ni) Department of Applied Mathematics, Nanjing Audit University, Nanjing 211815, People's Republic of China

E-mail address: nihexia@yeah.net 